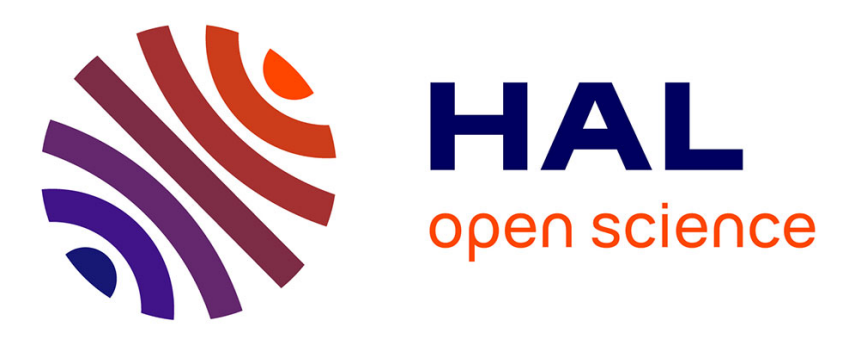

\title{
Large Neighborhood Local Search Optimization on Graphics Processing Units
}

Thé Van Luong, Nouredine Melab, El-Ghazali Talbi

\section{To cite this version:}

Thé Van Luong, Nouredine Melab, El-Ghazali Talbi. Large Neighborhood Local Search Optimization on Graphics Processing Units. Workshop on Large-Scale Parallel Processing (LSPP) in Conjunction with the International Parallel \& Distributed Processing Symposium (IPDPS), 2010, Atlanta, United States. inria-00520465

\section{HAL Id: inria-00520465 https://hal.inria.fr/inria-00520465}

Submitted on 23 Sep 2010

HAL is a multi-disciplinary open access archive for the deposit and dissemination of scientific research documents, whether they are published or not. The documents may come from teaching and research institutions in France or abroad, or from public or private research centers.
L'archive ouverte pluridisciplinaire HAL, est destinée au dépôt et à la diffusion de documents scientifiques de niveau recherche, publiés ou non, émanant des établissements d'enseignement et de recherche français ou étrangers, des laboratoires publics ou privés. 


\title{
Large Neighborhood Local Search Optimization on Graphics Processing Units
}

\author{
Thé Van Luong, Nouredine Melab, El-Ghazali Talbi
}

\begin{abstract}
Local search (LS) algorithms are among the most powerful techniques for solving computationally hard problems in combinatorial optimization. These algorithms could be viewed as "walks through neighborhoods" where the walks are performed by iterative procedures that allow to move from a solution to another one in the solution space. In these heuristics, designing operators to explore large promising regions of the search space may improve the quality of the obtained solutions at the expense of a highly computationally process. Therefore, the use of graphics processing units (GPUs) provides an efficient complementary way to speed up the search. However, designing applications on GPU is still complex and error-prone. We provide a methodology to design and implement large neighborhood LS algorithms on GPU. Finding efficient mappings of the neighborhood structures onto the GPU threads organization is a challenging issue dealt with in this paper. The work has been experimented for binary problems by deploying multiple neighborhood structures. The obtained results are convincing both in terms of efficiency, quality and robustness of the provided solutions at run time.
\end{abstract}

\section{Keywords}

Metaheuristics, Local Search, Neighborhoods, Graphics Processing Units (GPU), GeneralPurpose Computing on Graphics Hardware, Permuted Perceptron Problem.

\section{INTRODUCTION}

Plenty of hard problems in a wide range of areas including engineering design, telecommunications, logistics, biology, etc., have been modeled and tackled successfully with optimization approaches such as metaheuristics (generic heuristics). Local search algorithms is a class of metaheuristics which handle with a single solution iteratively improved by exploring its neighborhood in the solution space. Fig. 1 gives a general model for LS algorithms. At each iteration, a set of neighboring solutions is generated and evaluated. The best of these candidate solutions is selected to replace the current solution. The process is iterated until a stopping criterion is satisfied. Common LS heuristics of the literature are hill climbing, simulated annealing, tabu search, iterative local search and variable neighborhood search. A state-of-the-art of LS algorithms can be found in [1].

The definition of the neighborhood is a required common step for the design of any LS algorithm. The neighborhood structure plays a crucial role in the performance of a LS method. Theoretical and experimental studies have shown that the increase of the neighborhood size may improve the effectiveness (quality of provided solutions) of the LS algorithms [2]. Nevertheless, as it is generally CPU time-consuming it is not often fully exploited in practice. Indeed, experiments with large neighborhood algorithms are often stopped without convergence being reached. That is the reason why, in designing LS methods, there is often a compromise between the size of the neighborhood to use and the computational complexity to explore it. As a consequence, in LS algorithms, there is often a reduction of the size of the explored neighborhood at the expense of the effectiveness. To deal with such issues, only the use of parallelism allows to design algorithms based on large neighborhoods. Nowadays, GPU computing is recognized as a powerful way to achieve high-performance on long-running scientific applications [3]. Designing LS algorithms based on large neighborhood structures for solving real-world optimization problems are good 


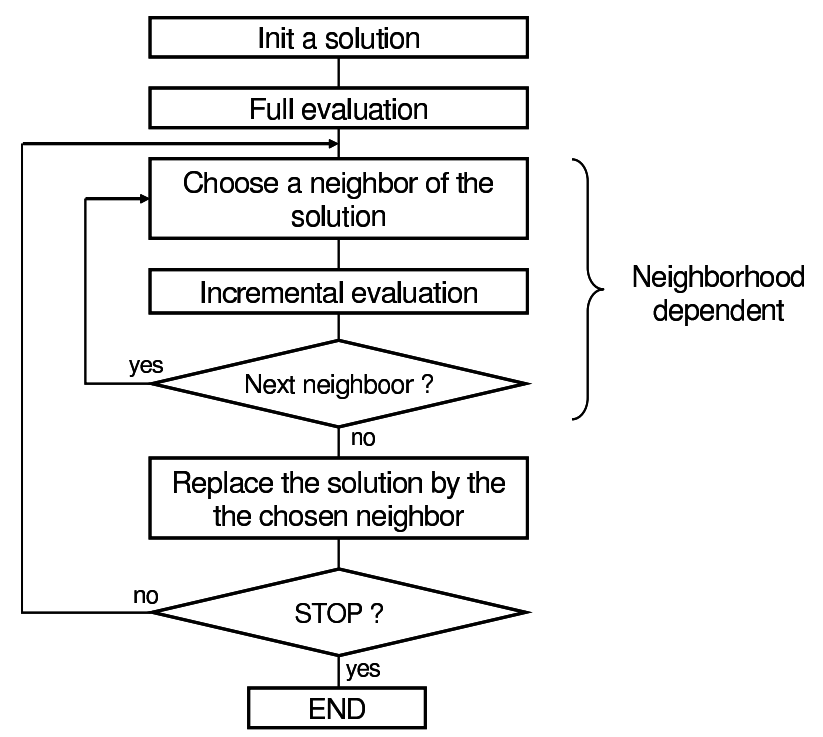

Fig. 1. General model for local search algorithms

challenges for GPU computing. However, to the best of our knowledge only few research works related to evolutionary algorithms on GPU exist [4]-[7]. Indeed, the parallel exploration of the neighborhood on GPU is not immediate and several challenges persist and are particular related to the characteristics and underlined issues of the GPU architecture and the LS algorithms.

In this paper, we contribute with the first results of LS algorithms based on large neighborhoods on GPU. The main objective of this paper is to find efficient mappings between the neighborhood structure and the hierarchical GPU. More exactly, the focus is on the mapping of the neighborhood of the currently processed solution to GPU threads. Since the neighborhood structure strongly depends on the target optimization problem, we focus on binary problems all along of this paper. We propose to deal with three neighborhoods of different sizes. For each handled neighborhood, the mappings of the neighborhood structure to the GPU thread blocks organization is particularly challenging.

To be valided the work has been experimented on the permuted perceptron problem (PPP) introduced by Pointcheval [8]. The problem is a cryptographic identification scheme based on NP-complete problems, which seems to be well suited for resource constrained devices such as smart cards. The work has been experimented on different popular instances of the literature. We investigate to measure the impact on how the increase of the size of the neighborhood can improve the quality of the obtained solutions.

The rest of the paper is organized as follows: Section 2 presents the three handled neighborhoods for binary problems. In Section 3, efficient mappings for each neighborhood structures are performed on GPU. Application of this methodology is made for the permuted perceptron problem in Section 4. Finally, conclusions and a discussion of this work are drawn in Section 5.

\section{NeIghborhoOdS FOR BINARY PROBLEMS}

Designing any iterative metaheuristic needs an encoding of a solution. The encoding must be suitable and relevant to the tackled optimization problem. For binary problems, any candidate solution is represented by a vector (or string) of binary values. Moreover, the efficiency of a representation is related to the search operators applied on this representation i.e. the neighborhood. 


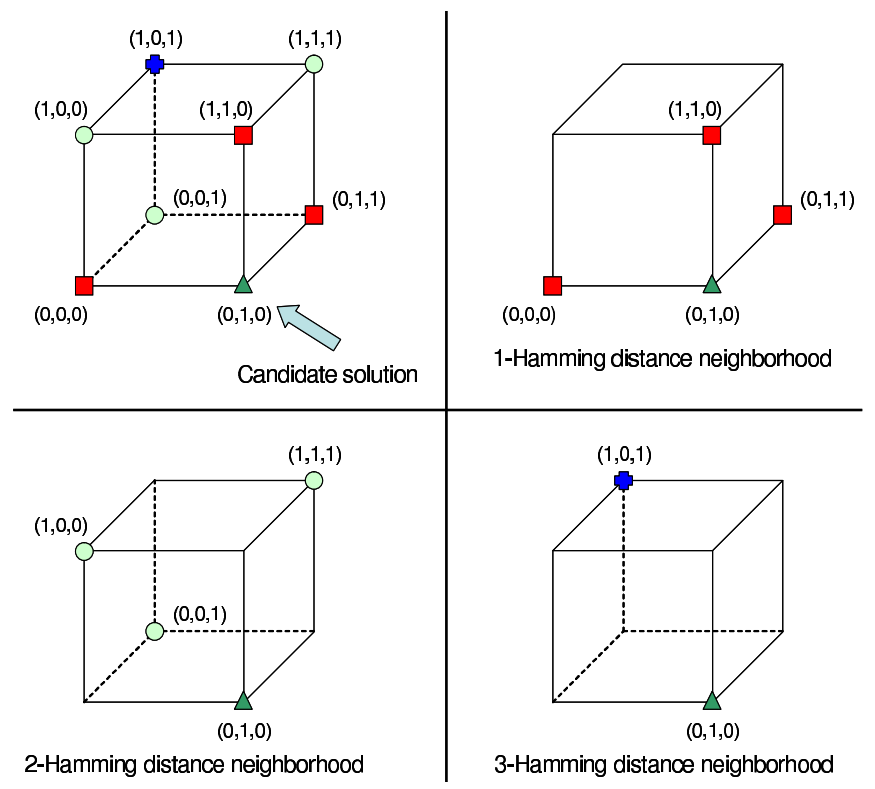

Fig. 2. Three neighborhoods for binary problems

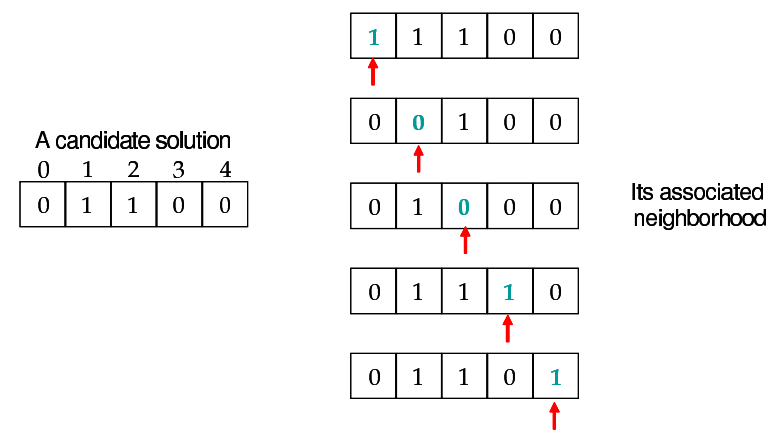

Fig. 3. 1-Hamming Distance Neighborhood

The natural neighborhood for binary representations is based on the Hamming distance. This distance measures the number of positions between two strings of equal length in which the corresponding symbols are different. Fig. 2 gives an illustration of the Hamming distance for strings of length 3 . For instance, the Hamming distance between the node $(0,1,0)$ (represented by a triangle) and each node represented by a circle is equal to two. Therefore, nodes of a same shape in the graph constitute a particular neighborhood of the node $(0,1,0)$.

- 1-Hamming Distance Neighborhood. In most cases, the associated neighborhood for binary representations is based on the Hamming distance equal to one. In this neighborhood, generate a neighbor consists in flipping one bit of the candidate vector solution (see Fig. 3). Considering a candidate vector solution of size $n$, the size of the associated neighborhood is $n$.

- 2-Hamming Distance Neighborhood. For binary problems, an improved neighborhood for LS algorithms is based on the Hamming distance of two. It consists on building a neighbor by flipping two values of a candidate solution vector. Two indexes represent a particular neighbor. For a candidate solution of size $n$, the number of neighbors is $\frac{n \times(n-1)}{2}$. Fig. 4 

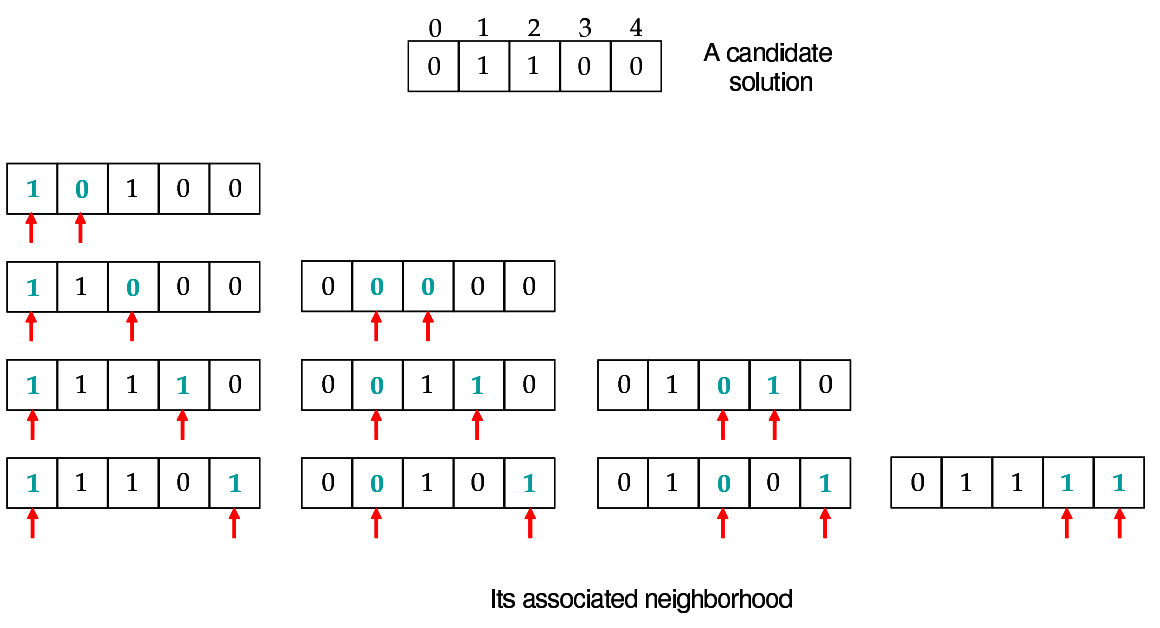

Fig. 4. 2-Hamming distance neighborhood

$$
\begin{array}{|l|l|l|l|l|}
\multicolumn{1}{c}{0} & 1 & 2 & 3 & 4 \\
0 & 1 & 1 & 0 & 0
\end{array} \quad \begin{gathered}
\text { A candidate } \\
\text { solution }
\end{gathered}
$$

\begin{tabular}{l|l|l|l|l|}
\hline 1 & 0 & 0 & 0 & 0 \\
\hline$\uparrow$ & $\uparrow$ & $\uparrow$ \\
\hline 1 & 0 & 1 & 1 & 0 \\
\hline$\uparrow$ & $\uparrow$ & \multicolumn{5}{|}{$\uparrow$} \\
\hline 1 & 0 & 1 & 0 & 1 \\
\hline$\uparrow$ & $\uparrow$ & \multicolumn{5}{c}{$\uparrow$}
\end{tabular}

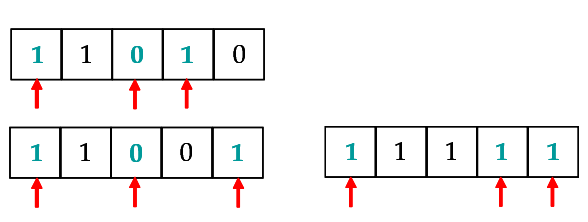

Its associated

neighborhood
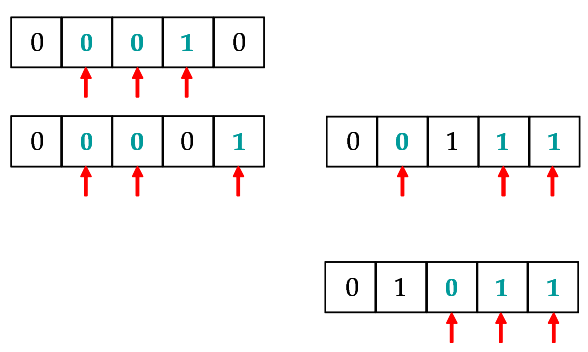

Fig. 5. 3-Hamming distance neighborhood

gives an illustration of this neighborhood.

- 3-Hamming Distance Neighborhood. An instance of a large neighborhood is a neighborhood built by modifying three values called 3 -Hamming distance neighborhood. This neighborhood is much complex since each neighboring solution is identified by 3 indexes. The number of elements associated to this neighborhood is $\frac{n \times(n-1) \times(n-2)}{6}$. Fig. 5 shows an illustration of this neighborhood.

Most of the LS algorithms use neighborhoods which are in general a linear (e.g. 1-Hamming distance) or quadratic (e.g. 2-Hamming distance) function of the input instance size. Some large neighborhoods may be high-order polynomial of the size of the input instance (e.g. 3Hamming distance). Then, the complexity of the search will be much higher. So, in practice, large neighborhoods algorithms are unusable because of their high computational cost. In the 
other sections, we will show how the use of GPU computing allows to fully exploit parallelism in such algorithms.

\section{EFFICIENT MAPPINGS OF NeIGHBORHOOdS StRUCTURES ON GPU}

In this section, the focus is made on the neighborhood generation on GPU. Indeed, this step is crucial in the design of new large neighborhood LS algorithms for binary problems since it is clearly identified as the gateway between a GPU process and a candidate neighbor.

\section{A. GPU Kernel Execution Model}

Each processor device on GPU supports the single program multiple data (SPMD) model, i.e. multiple autonomous processors simultaneously execute the same program on different data. For achieving this, the concept of kernel is defined. The kernel is a function callable from the host and executed on the specified device simultaneously by several processors in parallel.

This kernel handling is dependent of the general-purpose language. For instance, CUDA (Compute Unified Device Architecture) is a parallel computing environment, which provides an application programming interface for NVIDIA architectures [9]. The concept of thread in CUDA does not have exactly the same meaning as CPU thread. A thread on GPU can be seen as an element of the data to be processed. Compared to CPU threads, CUDA threads are lightweight. That means that changing the context between two threads is not a costly operation.

Regarding their spatial organization, threads are organized within so called thread blocks. A kernel is executed by multiple equally threaded blocks. Blocks can be organized into a onedimensional or two-dimensional grid of thread blocks, and threads inside a block are grouped in a similar way. All the threads belonging to the same thread block will be assigned as a group to a single multiprocessor, while different thread blocks can be assigned to different multiprocessors. Thus, a unique $i d$ can be deduced for each thread to perform computation on different data.

\section{B. Efficient mappings}

As suggested in Fig. 6, the challenging issue is to find efficient mappings between a thread $i d$ and a particular neighbor. Indeed, on the one hand, the thread $i d$ is represented by a single index. On the other hand, the move representation of a neighbor varies according to the neighborhood.

1) 1-Hamming Distance: For neighborhoods based on a Hamming distance of one, a mapping between LS neighborhood encoding and GPU threads is quiet direct. Indeed, for a binary vector of size $n$, the neighborhood size is exactly $n$ where each neighbor is represented by one index varying from 0 to $n-1$. Regarding the GPU threads, they are provided with a unique $i d$ and thus associated with one single index in a similar manner. That way, the associated kernel can be launched with $n$ threads (each neighbor is associated to a single thread). As a result, a $\mathbb{N} \rightarrow \mathbb{N}$ mapping can be made in constant time.

2) 2-Hamming Distance: For a binary vector of size $n$, the size of this new neighborhood is $\frac{n \times(n-1)}{2}$. The associated kernel is executed by $\frac{n \times(n-1)}{2}$ threads. For this encoding a mapping between a neighbor and a GPU thread is not straightforward. Indeed, on the one hand, a neighbor is composed by two indexes to modify. On the other hand, threads are identified by a unique $i d$ (single index). As a result, a $\mathbb{N} \rightarrow \mathbb{N} \times \mathbb{N}$ mapping has to be considered to transform one index into two. In a similar way, a $\mathbb{N} \times \mathbb{N} \rightarrow \mathbb{N}$ mapping must be handled to transform two indexes into one.

\section{Proposition 1: Two-to-one index transformation}




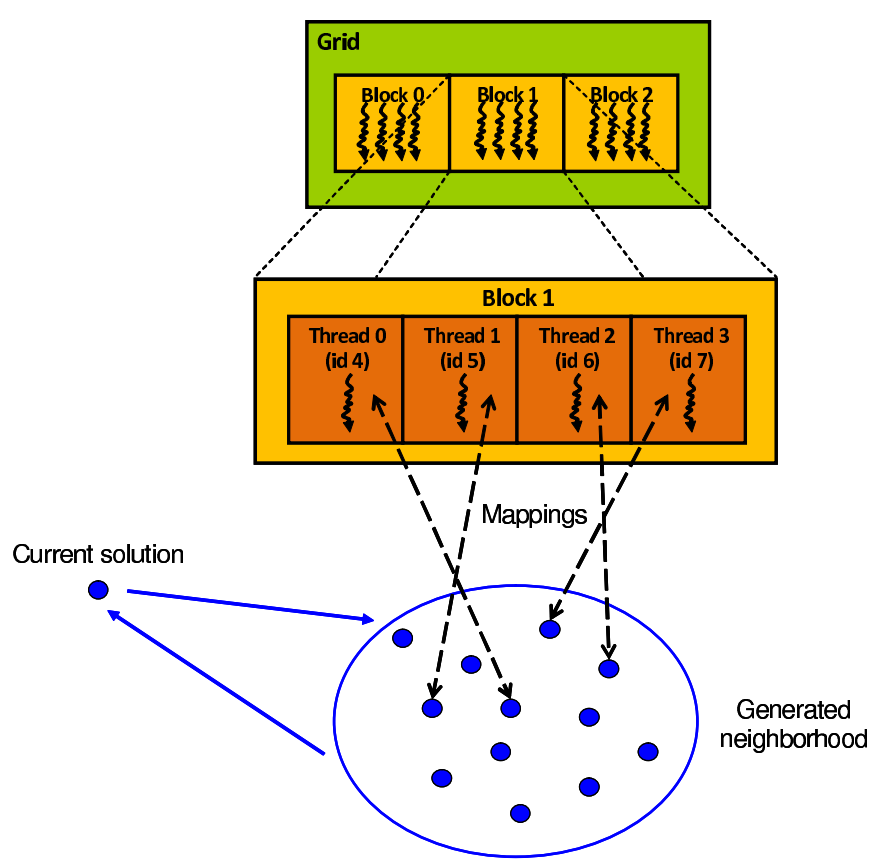

Fig. 6. Mappings between threads and neighbors

Given $i$ and $j$ the indexes of two elements to modify in the binary representation, the corresponding index $f(i, j)$ in the neighborhood representation is equal to $i \times(n-1)+(j-1)-\frac{i \times(i+1)}{2}$, where $n$ is the vector size.

\section{Proposition 2: One-to-two index transformation}

Given $f(i, j)$ the index of the element in the neighborhood representation, the corresponding index $i$ is equal to $n-2-\left\lfloor\frac{\sqrt{8 \times(m-f(i, j)-1)+1}-1}{2}\right\rfloor$ and $j$ is equal to $f(i, j)-i \times(n-1)+\frac{i \times(i+1)}{2}+1$ in the binary representation, where $n$ is the vector size and $m$ the neighborhood size.

The proofs of one-to-two and two-to-one index transformations are respectively in appendices $\mathrm{B}$ and A. The complexity of such mappings is dependent of the calculation of the square root on GPU (nearly constant time).

3) 3-Hamming Distance: For an array of size $n$, the size of this neighborhood is $\frac{n \times(n-1) \times(n-2)}{6}$. The associated kernel on GPU is executed by $\frac{n \times(n-1) \times(n-2)}{6}$ threads. A mapping here between a neighbor and a GPU thread is also particularly challenging. $\mathbb{N} \rightarrow \mathbb{N} \times \mathbb{N} \times \mathbb{N}$ and $\mathbb{N} \times \mathbb{N} \times \mathbb{N} \rightarrow$ $\mathbb{N}$ mappings must be handled efficiently.

The mapping here is a generalization of the 2-Hamming distance neighborhood with a third index. In this case, a 3D abstraction must be considered. For the sake of simplicity, instead of having a 3D view, we consider a set of plans where each plan is a $2 \mathrm{D}$ abstraction. The main difference with the 2D abstraction is the introduction of a third index which represents a plan in the $3 \mathrm{D}$ abstraction.

A methodology to perform one-to-three and three-to-one index transformations is given in appendices $\mathrm{C}$ and $\mathrm{D}$. The complexity of the mappings are logarithmic in practice (complexity of the numerical Newton-Raphson method). 


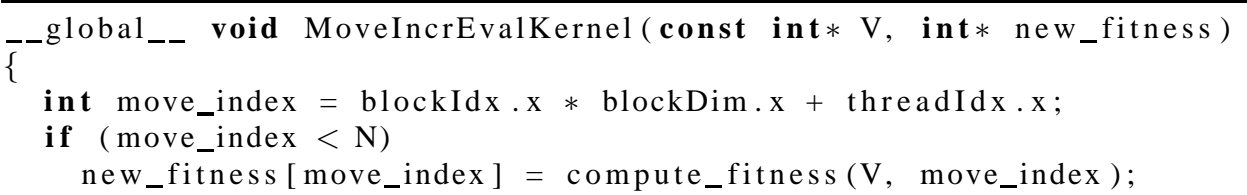

Fig. 7. Mapping source code for a neighborhood based on a Hamming distance of one

\section{Application to the Permuted Perceptron Problem}

\section{A. Permuted Perceptron Problem}

As illustration of a binary problem, the PPP is a NP-complete problem that has received a great attention given its importance in security protocols. An $\epsilon$-vector is a vector with all entries being either +1 or -1 . Similarly an $\epsilon$-matrix is a matrix in which all entries are either +1 or -1 . The PPP is defined as follows:

Definition 1: Given an $\epsilon$-matrix $\mathrm{A}$ of size $m \times n$ and a multiset $\mathrm{S}$ of non-negative integers of size $m$, find an $\epsilon$-vector $\mathrm{V}$ of size $n$ such that $\left\{\left\{(A V)_{j} / j=\{1, \ldots, m\}\right\}\right\}=S$.

Let $Y=A V$ be a matrix-vector product. Determine a histogram vector $H$ over the integers such that $H_{i}=\#\left\{Y_{j}=i \mid j=1, \ldots, m\right\}$. Let $V^{\prime}$ denote the candidate for the secret key $V$, let $Y^{\prime}=A V^{\prime}$ and let $H_{i}^{\prime}$ denote the histogram vector of $Y^{\prime}$. Then an objective function is given in [10] by:

$$
f\left(V^{\prime}\right)=30 \times \sum_{i=1}^{m}\left(\left|\left(A V^{\prime}\right)_{i}\right|-\left(A V^{\prime}\right)_{i}\right)+\sum_{i=1}^{n}\left(\left|H_{i}-H_{i}^{\prime}\right|\right) .
$$

This corresponds to a minimization problem where a value $f\left(V^{\prime}\right)=0$ gives a successful solution to the problem.

\section{B. Configuration}

A tabu search [11] has been implemented on GPU for each neighborhood. This algorithm is an instance of the general LS model presented in introduction. Basically, this algorithm uses a tabu list (a short-term memory) which contains the solutions that have been visited in the recent past. More details of this algorithm are given in [11].

The used configuration is an Intel Xeon 8 cores $3 \mathrm{GHz}$ with a NVIDIA GTX 280 card. The number of multiprocessors of this card is equal to 32 and the constraints of memory alignment are relaxed in comparaison with the previous architectures (G80 series). Therefore, GTX 280 get better global memory performance.

The following experiments intend to measure the quality of the solutions for the instances of the literature addressed in [10]. A tabu search was executed 50 times with a maximum number

of $\frac{n \times(n-1) \times(n-2)}{6}$ iterations (stopping criterion). The tabu list size was arbitrary set to a $\frac{m}{6}$ where $m$ is the number of neighbors. The average value of the evaluation function (fitness) and its standard deviation (in subindex) were measured. The number of successful tries (fitness equal to zero) and the average number of iterations are also represented.

\section{1-Hamming Distance}

Table I reports the results for the tabu search based on the 1-Hamming distance neighborhood and Fig. 7 shows the code source of the associated mapping. In a short execution time, the algorithm was able to find few solutions for the instances $m=73, n=73$ (10 successful tries 
TABLE I

PERMUTED PERCEPTRON PROBLEM 1-HAMming Distance

\begin{tabular}{|c|c|c|c|c|c|}
\hline Problem & Fitness & \# iterations & \# solutions & CPU time & GPU time \\
\hline $73 \times 73$ & $10.3_{5.1}$ & 59184.1 & $10 / 50$ & $4 s$ & $9 s$ \\
\hline $81 \times 81$ & $10.8_{5.6}$ & 77321.3 & $6 / 50$ & $6 s$ & $13 s$ \\
\hline $101 \times 101$ & $20.2_{14.1}$ & 166650 & $0 / 50$ & $16 s$ & $33 s$ \\
\hline $101 \times 117$ & $16.4_{5.4}$ & 260130 & $0 / 50$ & $29 s$ & $57 s$ \\
\hline
\end{tabular}

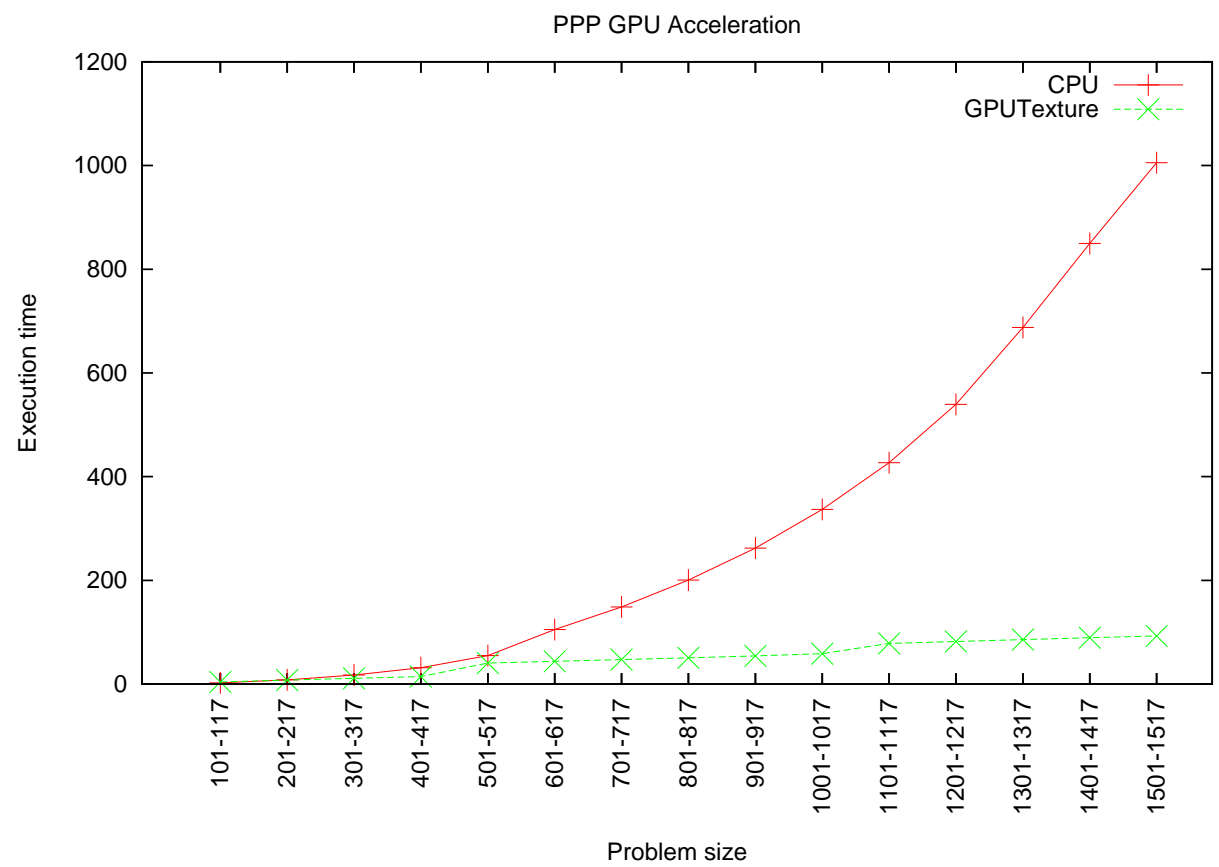

Fig. 8. GPU acceleration factor on the permuted perceptron problem

on 50) and $m=81, n=81$ (6 successful tries on 50). The two other instances are well-known for their difficulties and no solutions were found. Regarding execution time, GPU version does not offer anything in terms of efficiency. Indeed, since the neighborhood is relatively small $(n$ threads), the number of threads per block is not enough to fully cover the memory access latency.

To measure the efficiency of the GPU-based implemententation of this neighborhood, bigger instances of the PPP must be considered. Fig. 8 shows the GPU acceleration factor for different PPP instance sizes on the base of 10000 iterations.

From $m=201$ and $n=217$, the GPU version starts to be faster than CPU version (acceleration factor of $\times 1.1$ ). As long as the problem size increases, the speed-up grows significantly (up to $\times 10.8$ for $m=1501$ and $n=1517)$.

\section{2-Hamming Distance}

A tabu search has been implemented on GPU using a 2-Hamming distance neighborhood. The source code of the mapping is given in Fig. 9. Results of the experiment for the PPP are reported in Table II.

By using this other neighborhood, in comparison with Table I, the quality of solutions was significantly improved: on the one side the number of successful tries for both $m=73, n=73$ 


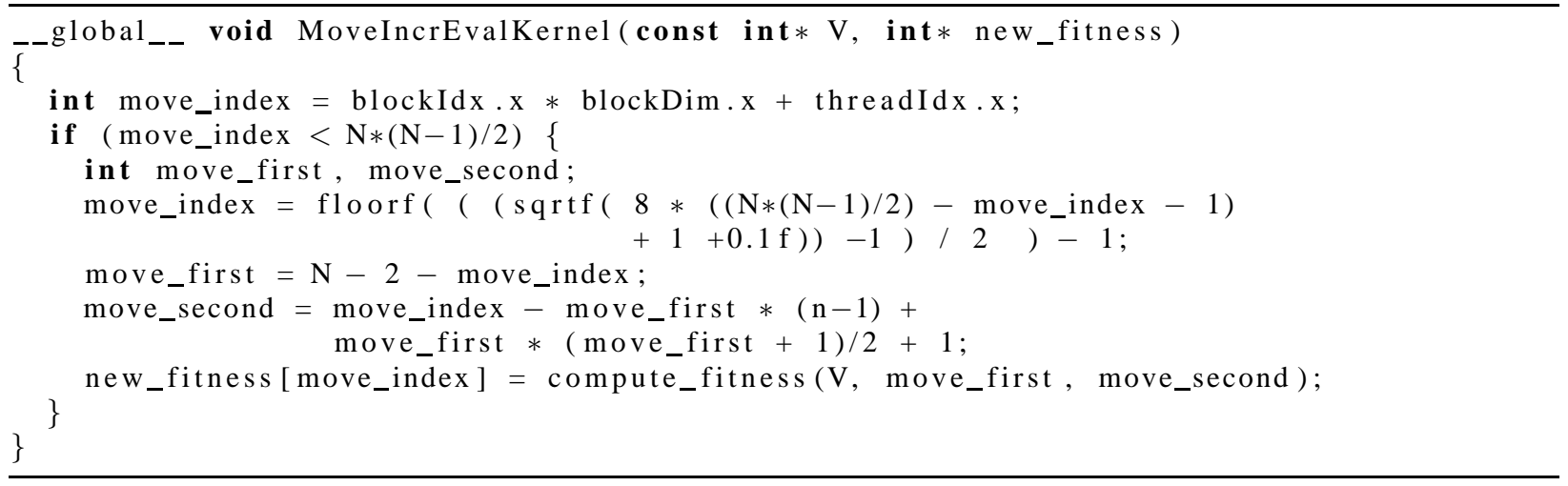

Fig. 9. Mapping source code for a neighborhood based on a Hamming distance of two

TABLE II

PERMUTED PERCEPTRON PROBLEM 2-HAMMING DisTANCE

\begin{tabular}{|c|c|c|c|c|c|c|}
\hline Problem & Fitness & \# iterations & \# solutions & CPU time & GPU time & Acceleration \\
\hline $73 \times 73$ & $16.4_{17.9}$ & 43031.7 & $19 / 50$ & $81 s$ & $8 s$ & $\times 9.9$ \\
\hline $81 \times 81$ & $15.5_{16.6}$ & 67462.5 & $13 / 50$ & $174 s$ & $16 s$ & $\times 11.0$ \\
\hline $101 \times 101$ & 14.214 .3 & 138349 & $12 / 50$ & $748 s$ & $44 s$ & $\times 17.0$ \\
\hline $101 \times 117$ & $13.8_{10.8}$ & 260130 & $0 / 50$ & $1947 s$ & $105 s$ & $\times 18.5$ \\
\hline
\end{tabular}

(19 solutions) and $m=81, n=81$ (13 solutions) is more important. On the other side, 12 solutions were found for the instance $m=101, n=101$. Regarding execution time, acceleration factor for GPU version is really efficient (from $\times 9.9$ to $\times 18.5$ ). Indeed, since a large number of threads are executed, GPU can take full advantage of the multiprocessors occupancy.

\section{E. 3-Hamming Distance}

A tabu search using a 3-Hamming distance neighborhood was implemented for the PPP. Fig. 10 shows a part of the source code for the mapping. Since the computational time was too exhorbitant, the average expected time for the CPU implementation was deduced from the base of 100 iterations per execution. Results are collected in Table III.

In comparison with Knudsen and Meier article [10], the results found by the generic tabu search are competitive without any use of cryptoanalysis techniques. Indeed, the number of successful solutions was drastically improved for every instance (respectively 35, 28 and 18 successful tries) and a solution was even found for the instance $m=101, n=117$. Regarding

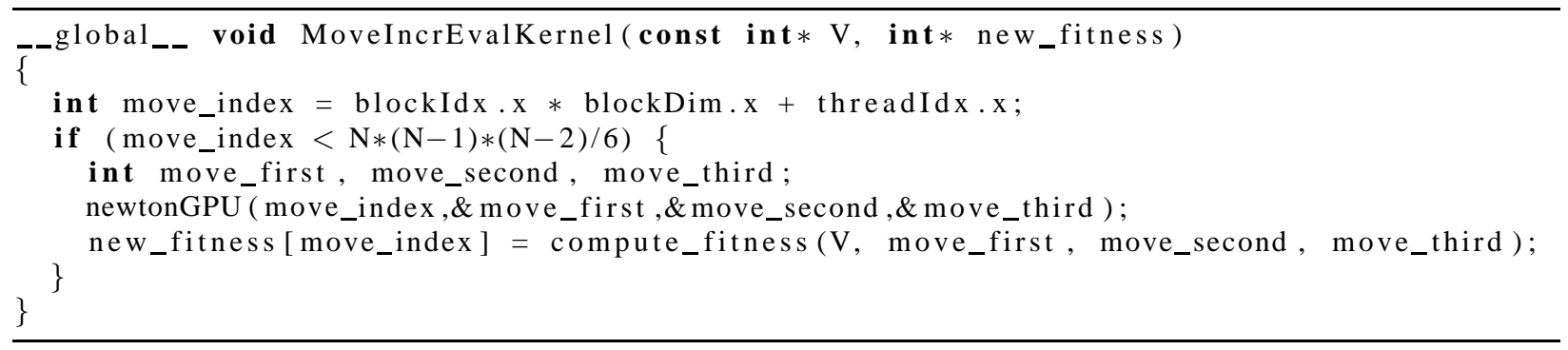

Fig. 10. Mapping source code for a neighborhood based on a Hamming distance of three 
TABLE III

PERMUTED PERCEPTRON PROBLEM 3-HAMMING DistANCE

\begin{tabular}{|c|c|c|c|c|c|c|}
\hline Problem & Fitness & \# iterations & \# solutions & CPU expected time & GPU time & Acceleration \\
\hline $73 \times 73$ & $2.4_{4.3}$ & 21360.2 & $35 / 50$ & $1202 \mathrm{~s}$ & $50 \mathrm{~s}$ & $\times 24.2$ \\
\hline $81 \times 81$ & $3.5_{4.4}$ & 43230.7 & $28 / 50$ & $3730 \mathrm{~s}$ & $146 \mathrm{~s}$ & $\times 25.5$ \\
\hline $101 \times 101$ & $6.2_{5.4}$ & 117422 & $18 / 50$ & $24657 \mathrm{~s}$ & $955 \mathrm{~s}$ & $\times 25.8$ \\
\hline $101 \times 117$ & $7.7_{2.7}$ & 255337 & $1 / 50$ & $88151 \mathrm{~s}$ & $3551 \mathrm{~s}$ & $\times 24.8$ \\
\hline
\end{tabular}

execution time, acceleration factors using GPU are very significant (from $\times 24.2$ to $\times 25.8$ ).

The conclusion from this experiment indicate that the use of GPU provides an efficient way to deal with large neighborhoods. Indeed, 3 Hamming-distance neighborhood on PPP were unpracticable in terms of single CPU computational ressources. So, implementing this algorithm on GPU has allowed to exploit parallelism in such neighborhood and improve the quality of solutions.

\section{Discussion AND CONCLUSION}

Local search algorithms based on large neighborhoods may allow to enhance the effectiveness in combinatorial optimization [2]. However, their exploitation for solving real-world problems is possible only by using a great computing power. High-performance computing based on GPU accelerators is recently revealed as an efficient way to use the huge amount of resources at disposal and fully exploit the parallelism of neighborhoods. To the best of our knowledge, no research work has been published on LS algorithms on GPU based on different neighborhoods exploration.

In this paper, we particularly focused on the design of efficient mappings of three different neighborhoods to the hierarchical GPU for binary problems. The designed and implemented approaches have been experimentally validated on a cryptographic application. The experiments indicate that GPU computing allows not only to speed up the search process, but also to exploit large neighborhoods structures to improve the quality of the obtained solutions. For instance, LS algorithms based on a Hamming distance of three were unpracticable on traditional machines because of their high computational cost. So, GPU computing has permitted their achievement and the obtained results are particularly promising in terms of effectiveness. Indeed, all along the paper, we investigated on how the increase of the size of neighborhood allows to improve the quality of the solutions. Furthermore, we strongly believe that the quality of the solutions would be drastically enhanced by (1) increasing the number of running iterations of the algorithm and (2) introducing appropriate cryptonanalysis heuristics.

Beyond the improvement of the effectiveness, the parallelism of GPUs allows to push far the limits in terms of computational resources. As a consequence, a next perspective is to use a multi-GPU approach to allow handling larger neighborhoods. It will consist of partitioning the neighborhood set, where each partition is executed on a single GPU. That way, multi-GPU approach will allow to increase the speed-up of the exploration space of a given solution. But since each GPU has its own private memory, managing the context execution of different GPUs in an efficient way is not a straighforward task.

In the future, GPU concepts will be integrated in the ParadisEO platform. This framework was developped for the design of parallel hybrid metaheuristics dedicated to the mono/multiobjective resolution [12]. ParadisEO can be seen as a white-box object-oriented framework based on a clear conceptual separation of metaheuristics concepts. The Parallel Evolving Objects (PEO) module 


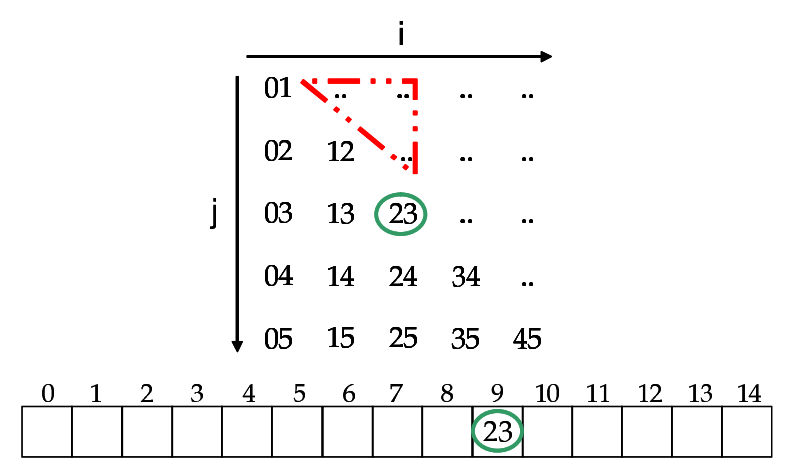

Fig. 11. $\quad \mathbb{N} \times \mathbb{N} \rightarrow \mathbb{N}$ mapping

of ParadisEO includes the well-known parallel and distributed models for metaheuristics. This module will be extended in the future with GPU-based implementation.

\section{APPENDIX A}

\section{TWO-TO-ONE INDEX TRANSFORMATION}

Let us consider a 2D abstraction in which elements of the neighborhood are disposed in a zero-based indexing 2D representation in a similar way that a lower triangular matrix. Let $n$ be the size of the solution representation and let $m=\frac{n \times(n-1)}{2}$ be the size of its neighborhood. Let $i$ and $j$ be the indexes of two elements to modify in a binary encoding. A candidate neighbor is then identified by both $i$ and $j$ indexes in the 2D abstraction. Let $f(i, j)$ be the corresponding index in the 1D neighborhood fitnesses structure. Fig. 11 gives through an example an illustration of this abstraction.

In this example, $n=6, m=15$ and the neighbor identified by the coordinates $(i=2, j=3)$ is mapped to the corresponding $1 \mathrm{D}$ array element $f(i, j)=9$.

The neighbor represented by the $(i, j)$ coordinates is known, and its corresponding index $f(i, j)$ on the 1D structure has to be calculated. If the 1D array size was $n * n$, the $2 \mathrm{D}$ abstraction would be similar to a matrix and the $\mathbb{N} \times \mathbb{N} \rightarrow \mathbb{N}$ mapping would be:

$$
f(i, j)=i \times(n-1)+(j-1)
$$

Since the 1D array size is $m=\frac{n \times(n-1)}{2}$, in the 2D abstraction, elements above the diagonal preceding the neighbor must not be considered (illustrated in Fig. 11 by a triangle). The corresponding mapping $\mathbb{N} \times \mathbb{N} \rightarrow \mathbb{N}$ is therefore:

$$
f(i, j)=i \times(n-1)+(j-1)-\frac{i \times(i+1)}{2}
$$

\section{APPENDIX B}

\section{ONE-TO-TWO INDEX TRANSFORMATION}

Let us consider the $2 \mathrm{D}$ abstraction previously presented. If the element corresponding to $f(i, j)$ in the 2D abstraction has a given $i$ abscissa, then let $k$ be the distance plus one between the $i+1$ and $n-2$ abscissas. If $k$ is known, the value of $i$ can be deduced:

$$
i=n-2-\left\lfloor\frac{\sqrt{8 X+1}-1}{2}\right\rfloor
$$




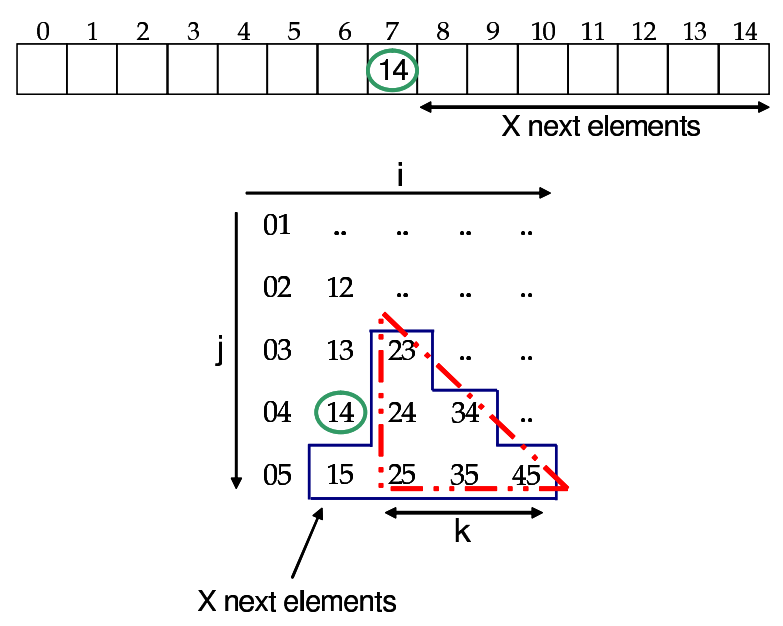

Fig. 12. $\quad \mathbb{N} \rightarrow \mathbb{N} \times \mathbb{N}$ mapping

Let $X$ be the number of elements following $f(i, j)$ in the neighborhood index-based array numbering:

$$
X=m-f(i, j)-1
$$

Since this number can be also represented in the $2 \mathrm{D}$ abstraction, the main idea is to maximize the distance $k$ such as:

$$
\frac{k \times(k+1)}{2} \leq X
$$

Fig. 12 gives an illustration of this idea (represented by a triangle).

Resolving (4) gives the greatest distance $k$ :

$$
k=\left\lfloor\frac{\sqrt{8 X+1}-1}{2}\right\rfloor
$$

A value of $i$ can then be calculated according to (2). Finally, by using (1) $j$ can be given by:

$$
j=f(i, j)-i \times(n-1)+\frac{i \times(i+1)}{2}+1
$$

$\mathbb{N} \rightarrow \mathbb{N} \times \mathbb{N}$ mapping is also done.

\section{APPENDIX C}

ONE-TO-THREE INDEX TRANSFORMATION

$f(x, y, z)$ is a given index of the 1D neighborhood fitnesses structure and the objective is to find the three indexes $x, y$ and $z$. Let $n$ be the size of the solution representation and $m=\frac{n \times(n-1) \times(n-2)}{6}$ be the size of the neighborhood. The main idea is to find in which plan (coordinate $z$ ) corresponds the given element $f(x, y, z)$ in the $3 \mathrm{D}$ abstraction. If this corresponding plan is found, then the rest is similar as the $\mathbb{N} \rightarrow \mathbb{N} \times \mathbb{N}$ mapping for the one-to-two index transformation previously seen. Figure 13 illustrates an example of the 3D abstraction.

In this representation, since each plan is a $2 \mathrm{D}$ abstraction, the number of elements in each plan is the number of combinations $\left(\begin{array}{l}k \\ 2\end{array}\right)$ where $k \in\{2,3, \ldots, n-1\}$ according to each plan. For a specific neighbor, if a value of $k$ is found, then the value of the corresponding plan $z$ is:

$$
z=n-k-1
$$




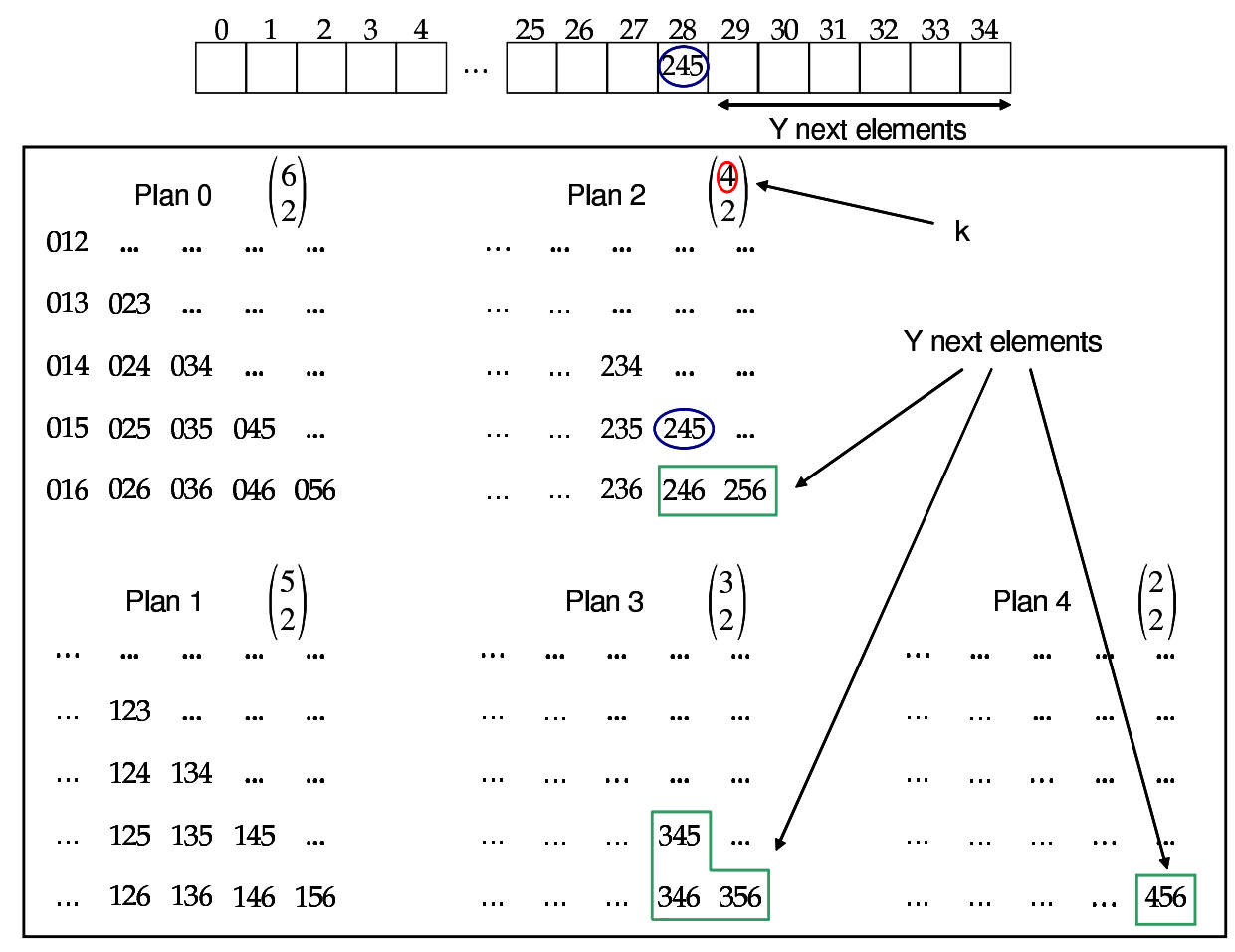

Fig. 13. $\quad \mathbb{N} \rightarrow \mathbb{N} \times \mathbb{N} \times \mathbb{N}$ mapping

For a given index $f(x, y, z)$ belonging to the plan $k$ in the $3 \mathrm{D}$ abstraction, the number of elements contained in the following plans is $\left(\begin{array}{l}k \\ 3\end{array}\right)$ (also equal to $\frac{k \times(k-1) \times(k-2)}{6}$ ).

Let $Y$ be the number of elements following $f(x, y, z)$ in both 1D neighborhood fitnesses structure and 3D abstraction:

$$
Y=m-f(x, y, z)
$$

Then the main idea is to minimize $k$ such as:

$$
\frac{k \times(k-1) \times(k-2)}{6}>=Y
$$

By reordering (8), in order to find a value of $k$, the next step is to solve the following equation:

$$
k_{1}^{3}-k_{1}-6 Y=0
$$

Cardano's method in theory allows to solve cubic equation. Nevertheless, in the case of finite discrete machine, this method can lose precision especially for big integers. As a consequence, a simple Newton-Raphson method for finding an approximate value of $k_{1}$ is enough for our problem. Indeed, this iterative process follows a set guideline to approximate one root, considering the function, its derivative, an initial arbitrary $k_{1}$-value and a certain precision (see Algorithm 1).

Finally, since the minimization of $k$ in (8) is expected, the value of $k$ is:

$$
k=\left\lceil k_{1}\right\rceil
$$

Then a value of $z$ can be deduced with (7). At this step, the plan corresponding to the element $f(x, y, z)$ is known. The next steps for finding $x$ and $y$ are identically the same as the one-to-two index transformation with a change of variables. 


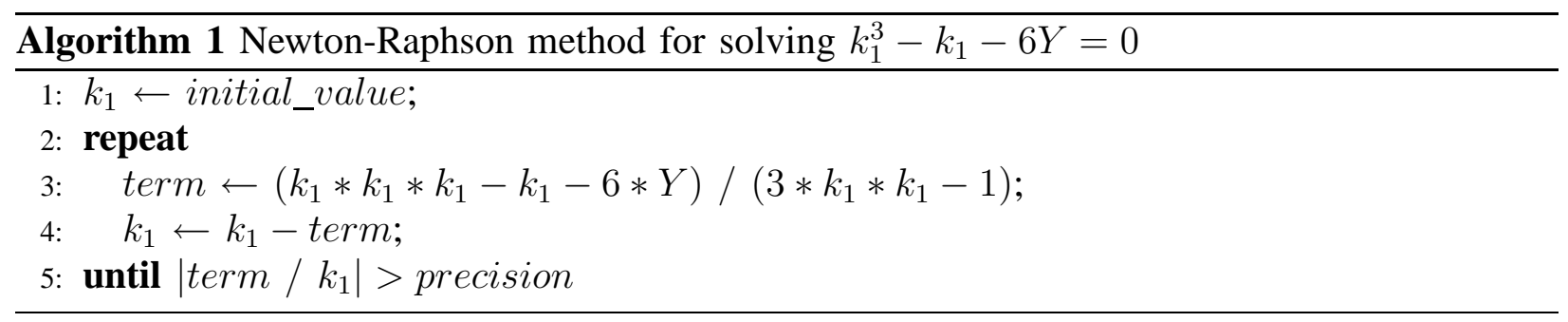

First, the number of elements preceding $f(x, y, z)$ in the neighborhood index-bas array numbering is exactly:

$$
n b \text { ElementsBefore }=m-\frac{(k+1) \times k \times(k-1)}{6}
$$

Second, the number of elements contained in the same plan $z$ as $f(x, y, z)$ is:

$$
\text { nbElements }=\frac{k \times(k-1)}{2}
$$

Finally the index of the last element of the plan $z$ is:

$$
\text { lastElement }=\text { nbElementsBefore }+ \text { nbElements }-1
$$

As a result, one-to-two index transformation is applied with a change of variables:

$$
\begin{gathered}
f(i, j)=f(x, y, z)-\text { nbElementsBefore } \\
n^{\prime}=n-(z+1) \\
X=\text { lastElement }-f(x, y, z)
\end{gathered}
$$

After performing this transformation, a value of $x$ and $y$ can be deduced:

$$
\begin{aligned}
& x=i+(z+1) \\
& y=j+(z+1)
\end{aligned}
$$

$\mathbb{N} \rightarrow \mathbb{N} \times \mathbb{N} \times \mathbb{N}$ mapping is done.

\section{APPENDIX D}

\section{THREE-TO-ONE INDEX TRANSFORMATION}

$x, y$ and $z$ are known and its corresponding index $f(x, y, z)$ must be found. According to the $3 \mathrm{D}$ abstraction, since a value of $z$ is known, $k$ can be calculated:

$$
k=n-1-z
$$

Then the number of elements preceding $f(x, y, z)$ in the neighborhood index-based array numbering can be also deduced.

If each plan size was $(n-2) *(n-2)$, each $2 \mathrm{D}$ abstraction would be similar to a matrix and the $\mathbb{N} \times \mathbb{N} \rightarrow \mathbb{N}$ mapping would be:

$$
f_{1}(x, y, z)=z \times(n-2) \times(n-2)+(x-1) \times(n-2)+(y-2)
$$




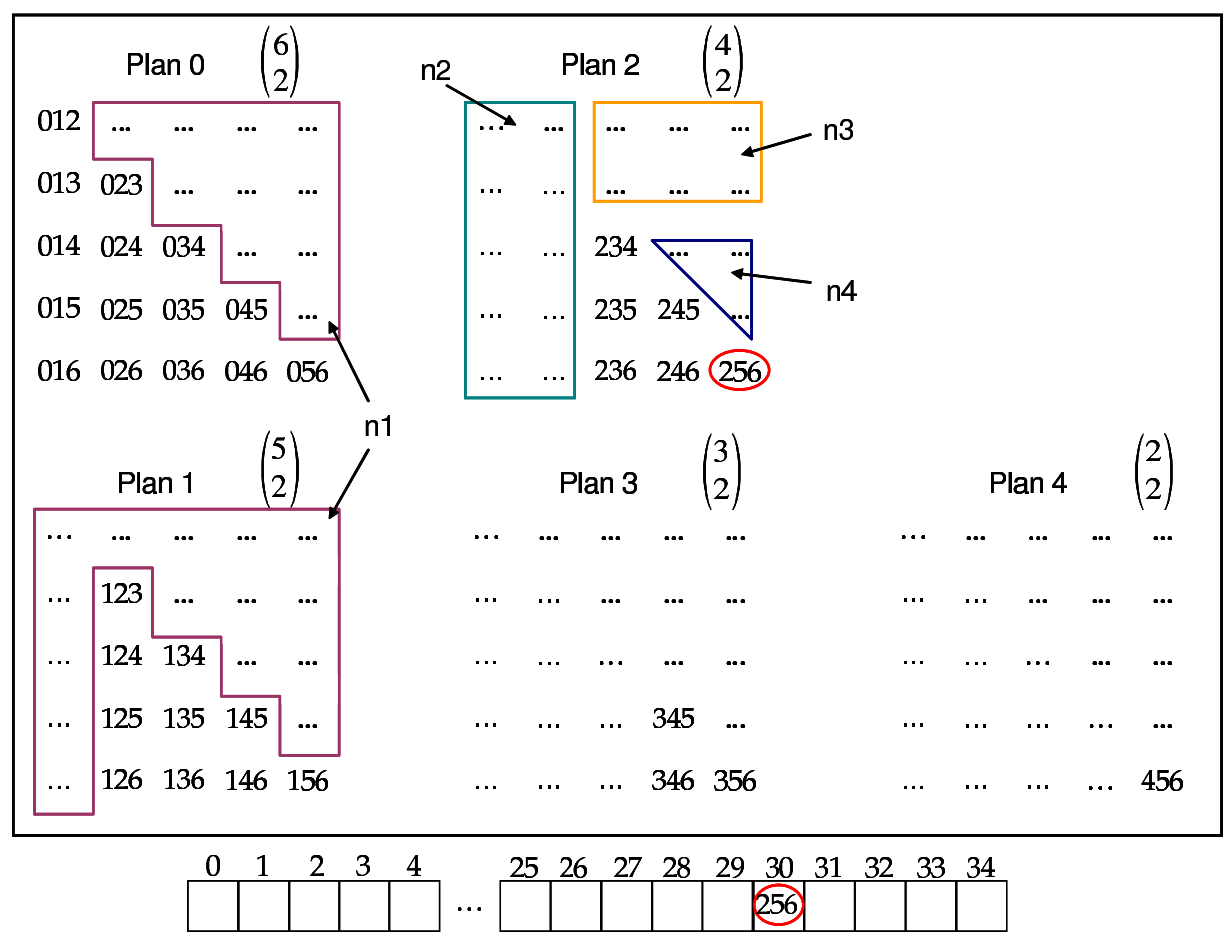

Fig. 14. $\quad \mathbb{N} \times \mathbb{N} \times \mathbb{N} \rightarrow \mathbb{N}$ mapping

Since each 2D abstraction is some kind of triangular matrix, some elements must not be considered. The advantage of the $3 \mathrm{D}$ abstraction is that these elements can be found by geometric construction (see Fig. 14).

First, given a plan $z$, the number of elements in the previous plans to not consider is:

$$
n 1=z \times(n-2) \times(n-2)-\text { nbElementsBefore }
$$

Second, the number of elements on the left side to not consider in the plan $z$ is:

$$
n 2=z \times(n-2)
$$

Third, the number of elements on the upper side to not consider in the plan $z$ is:

$$
n 3=(y-z) \times(n-k-1)
$$

Fourth, the number of elements on the upper triangle above $f(x, y, z)$ to not consider is:

$$
n 4=\frac{(y-z) \times(y-z-1)}{2}
$$

Finally a value of $f(x, y, z)$ can be deduced:

$$
f(x, y, z)=f_{1}(x, y, z)-n 1-n 2-n 3-n 4
$$

$\mathbb{N} \times \mathbb{N} \times \mathbb{N} \rightarrow \mathbb{N}$ mapping is also done. 


\section{REFERENCES}

[1] E.-G. Talbi, From design to implementation. Wiley, 2009.

[2] R. K. Ahuja, J. Goodstein, A. Mukherjee, J. B. Orlin, and D. Sharma, "A very large-scale neighborhood search algorithm for the combined through-fleet-assignment model," INFORMS Journal on Computing, vol. 19, no. 3, pp. 416-428, 2007.

[3] S. Ryoo, C. I. Rodrigues, S. S. Stone, J. A. Stratton, S.-Z. Ueng, S. S. Baghsorkhi, and W. mei W. Hwu, "Program optimization carving for gpu computing," J. Parallel Distrib. Comput., vol. 68, no. 10, pp. 1389-1401, 2008.

[4] J.-M. Li, X.-J. Wang, R.-S. He, and Z.-X. Chi, "An efficient fine-grained parallel genetic algorithm based on gpu-accelerated," in Network and Parallel Computing Workshops, 2007. NPC Workshops. IFIP International Conference, 2007, pp. 855-862. [Online]. Available: http://dx.doi.org/10.1109/NPC.2007.108

[5] D. M. Chitty, "A data parallel approach to genetic programming using programmable graphics hardware," in GECCO, 2007, pp. 1566-1573.

[6] T.-T. Wong and M. L. Wong, "Parallel evolutionary algorithms on consumer-level graphics processing unit," in Parallel Evolutionary Computations, 2006, pp. 133-155.

[7] K.-L. Fok, T.-T. Wong, and M. L. Wong, "Evolutionary computing on consumer graphics hardware," IEEE Intelligent Systems, vol. 22, no. 2, pp. 69-78, 2007.

[8] D. Pointcheval, "A new identification scheme based on the perceptrons problem," in EUROCRYPT, 1995, pp. 319-328.

[9] NVIDIA, CUDA Programming Guide Version 2.1, 2009

[10] L. R. Knudsen and W. Meier, "Cryptanalysis of an identification scheme based on the permuted perceptron problem," in EUROCRYPT, 1999, pp. 363-374.

[11] É. D. Taillard, "Robust taboo search for the quadratic assignment problem," Parallel Computing, vol. 17, no. 4-5, pp. 443-455, 1991.

[12] S. Cahon, N. Melab, and E.-G. Talbi, "Paradiseo: A framework for the reusable design of parallel and distributed metaheuristics," J. Heuristics, vol. 10, no. 3, pp. 357-380, 2004. 